\title{
Thermoregulatory responses of Baladi and crossbred goat does under subtropical conditions.
}

\author{
El-zaher ${ }^{1}$ H. M., El-Sayed ${ }^{2}$ A. I. M., Farghaly ${ }^{1}$ H. A. M. and Teama ${ }^{1}$ F. E. I.
}

1. Department of Biological Applications, Radioisotopes Applications Division, Nuclear Research Center, Atomic Energy Authority, Inshas, Cairo, Egypt, P.O.13759

2. Department of Animal Production, Faculty of Agriculture, Benha University, Cairo, Egypt, P.O.13736.

Corresponding author: hus_ahm2002@yahoo.com

\begin{abstract}
This experiment was conducted to study the effect of heat stress on thermoregulatory responses in Baladi and crossbrd (50\% Baladi x 50\% Zaraibi) female goats reared under desert of Inshas area during estrous cycle, pregnancy stages and postpartum periods under hot season (period from May, 2014 to October, 2014) compared with mild season (period from October, 2013 to March, 2014) and measure the difference between performance of crossbred animals and performance of the breed that were crossed to evaluate adaptability of two breed throughout these stages, point out hot climate condition of Egyptian environment. It has been used in this research 40 mature female goats (20 Baladi and 20 crossbred (50\% Zaraibi x 50\% Baladi) with an average age of 2-3 years and an average body weight was ranging from $25 \pm 1.5 \mathrm{~kg}$ to $35 \pm 1.6 \mathrm{~kg}$ for Baladi and crossbred, respectively, the experiment lasted for two seasons (mild and hot). The animals were kept in semi-open pens during the experimental period and allowed to graze for five hours daily at least. Thermoregulatory responses of the animal were estimated in terms of rectal temperature (RT), skin temperature (ST) and respiratory rate (RR).Results showed significant decrease $(\mathrm{P}<0.0001)$ in $\mathrm{RT}$, ST and RR during mild season as compared with hot season during the estrous cycle. Also, crossbred showed lower $(\mathrm{P}<0.01$ or $\mathrm{P}<0.05) \mathrm{RT}$ in comparison with Baladi goat does during different stages of estrous cycle. While ST and RR showed insignificant variations between crossbred and Baladi goat does during different stage of estrous cycle. During pregnancy stages Baladi and crossbred goat does recorded significant low $(\mathrm{P}<0.0001) \mathrm{RT}$, ST and RR under mild season compared with hot season throughout different stages of pregnancy. Crossbred showed lower $(\mathrm{P}<0.05$ or $\mathrm{P}<0.0001)$ RT than Baladi goat does during different stages of pregnancy, however insignificant variations in ST and RR between crossbred and Baladi goat does during different stages of pregnancy. During postpartum periods Baladi and crossbred goat does recorded significant low $(\mathrm{P}<0.05$ or $\mathrm{P}<0.0001)$ RT, ST and RR under mild season in comparison with hot season throughout different stages of postpartum. While RT showed significant variation $(\mathrm{P}<0.05)$ between crossbred and Baladi goat does in different stages of postpartum (day of parturition and 15 days postpartum), except 30 days postpartum which insignificantly affected by breed. ST showed significant variation $(\mathrm{P}<0.01)$ between crossbred and Baladi goat does in 30 days postpartum only. However RR showed significant $(\mathrm{P}<0.05$ or $\mathrm{P}<0.01)$ decrease in crossbred as compared with Baladi goat does in different stages of postpartum. From the present results, it could be concluded that, each of season, goat breed and physiological status were affected on rectal temperature, skin temperature and respiration rate throughout the estrous cycle, pregnancy stages and postpartum period of Baladi goat does and its crosses with Zaraibi (50\% Baladi x 50\% Zaraibi).
\end{abstract}

Key words: Heat stress, rectal temperature, skin temperature, respiration rate, Baladi, Crossbred, female goats

\section{Introduction}

The summer in Egypt, is characterized by high ambient temperature, intense solar radiation and high relative humidity. Therefore, farm animals raised in such sever climatic stress for almost 6 months of the year and become uncomfortable and they suffer extremely in production, reproduction and resistance to diseases and parasites (Marai et al., 2002). Heat stress has a reducing effect on both the productivity and reproductive efficiency of farm animals (Jordan, 2003). The deleterious effects of heat stress are the result of either the hyperthermia associated with heat stress or the physiological adjustments made by the heat-stressed animal to regulate body temperature (Hansen, 2009). Rectal temperature, skin temperature and respiration rate are parameters which illustrate the mechanism of physiological adaptation (Marai et al., 2007; Otoikhian et al., 2009; Phulia et al., 2010 and Sharma et al., 2013). Rectal temperature (RT) is considered as a good index of body temperature even though there is a considerable variation in different parts of the body core at different times of the day (Srikandakumar et al., 2003). Even a rise of less than $1^{\circ} \mathrm{C}$ in rectal temperature was enough to reduce performance in most livestock species which makes the body a sensitive indicator of physiological response to heat stress (Shebaita and El-Banna, 1982). Rectal temperature of goats was found to be elevated with high environmental temperature in several studies (Devendra, 1987; Marai et al., 2007). Exposure to 
heat stress increase skin temperatures in goats (Fahmy 1994; Marai et al., 1997). khalifa et al., (2000) showed that exposure of goats to solar radiation increased significantly $(\mathrm{p}<0.01)$ skin temperature by $1.0^{\circ} \mathrm{C}$ and respiration rate by $18 \mathrm{r} / \mathrm{min}$ During exposure to heat, ST was increased due to vasodilation which was beneficial for increasing heat loss from the skin (Slee, 1966). Also, Alam et al., (2011) stated that the respiration rate increased significantly $(\mathrm{P}<0.01)$ with increasing the period of exposure to heat stress (from 4 to $8 \mathrm{hrs}$. per day) compared with the control group $(0$ hrs heat exposure). A properly designed crossbreeding system allows the livestock producer to take advantage of appropriate combinations of the superior traits of several different breeds and it also yields heterosis which often referred to as hybrid vigor, measures the difference between average performance of crossbred animals and average performance of the breeds that were crossed to produce them (Olson, 2011). However, little information was available on performance of such introduced breeds and their crosses in Egypt, especially, under different climatic conditions.

The main objective of this study is to evaluate the effect of heat stress on thermoregulatory responses (rectal temperature, skin temperature and respiration rate) during hot season compared with mild season in each of native Baladi as purebred goat does and in its crosses with Zaraibi as crossbred goat does (50\% Zaraibi $\mathrm{x} 50 \%$ Baladi) reared under desert of Inshas area during estrous cycle, pregnancy stages and postpartum period to evaluate adaptability of two breed, point out hot climate condition of Egyptian environment.

\section{Materials and Methods}

\section{Experimental Animals:}

Forty native female goats, 20 Baladi and 20 crossbred (50\% Zaraibi x 50\% Baladi) were used. The does were selected according to reproductive farm records and subjected to the study. Their age ranged from 2 to 3 years old and the mean body weight for Baladi and crossbred was ranging from $25 \pm 1.5 \mathrm{~kg}$ to $35 \pm 1.6 \mathrm{~kg}$, respectively. All animals were healthy and clinically free of diseases.

\section{Animal Housing:}

All experimental goats were kept in semi-open pens throughout the period of experiment. These pens provided enough shade and ventilation in summer and protection from rain in winter. The does were allowed to graze five hours daily at least.

\section{Animal Feeding:}

The experiment was carried out in the Experimental Farms Project (Goats Farm), Nuclear Research Center, Atomic Energy Authority, Inshas. The experimental goats were fed basal ration of concentrate feed mixture (CFM) according to the allowances of NRC (2007) of goats. The CFM composed of $37.4 \%$ wheat bran, $27 \%$ yellow corn, $12.5 \%$ soybean meal, $10.0 \%$ undecorticated cottonseed cake, $5 \%$ rice bran, $4 \%$ sugarcane molasses, $3 \%$ limestone, $1 \%$ sodium chloride and 0.1 vitamin and minerals premix. Feed mixture was offered once daily at $10 \mathrm{am}, 3.5 \%$ of body weight. Barseem hay was offered ad libitum. Fresh drinking water was available at all time.

\section{Experimental measurements:}

Ambient temperature, relative humidity and temperature humidity index:

The ambient temperature and relative humidity were obtained daily from meteorological station of Atomic Energy Authority during the whole experimental period. The temperature humidity index (THI) was calculated during mild (period from October, 2013 to March, 2014) and hot (period from May, 2014 to October, 2014) seasons according to Marai et al., (2000) as:

$\mathrm{THI}=\mathrm{db}{ }^{\circ} \mathrm{C}-\left[(0.31-0.31 \mathrm{RH}) \times\left(\mathrm{db}{ }^{\circ} \mathrm{C}-14.4\right)\right]$

Where, $\mathrm{THI}=$ temperature humidity index, $\mathrm{db}$ ${ }^{\circ} \mathrm{C}=$ dry bulb temperature in Celsius and $\mathrm{RH}=$ relative humidity $\%$. A value for THI $<22.2$ was considered remarkably an absence of heat stress, while the values 22.2 to 23.3 referred to moderate (mild) heat stress, 23.3 to $<25.6$ referred to severe heat stress and $>25.6$ referred to very severe heat stress.

\section{Rectal and skin temperature:}

Rectal temperature (RT) was measured using digital clinical thermometer. Skin temperature (ST) was measured using $\mathrm{K}$ type sensor digital thermometer, model: 301 with measuring range: $-50^{\circ} \mathrm{C}$ to $1300^{\circ} \mathrm{C}$.

\section{Respiratory rate:}

Respiration rate (RR) was expressed as the number of respirations per minute (breaths/min.) and was measured by counting the flank movements in one minute. Complete inward and outward movement of the flank was counted as one respiration and was recorded.

\section{Statistical analysis}

Data were expressed as least square means \pm SE. Covariance analysis was carried out due to the significant differences found in the metabolic weight (initial live body weights ${ }^{0.75}$ ) of the experimental goat does (Atta, 2014). Data were statistically analyzed using procedure of SAS (1991) according the following model:

$Y_{i j k}=\mu+S_{i}+B_{j}+S_{i j}+b(X-\bar{x})+e_{i j k}$

Where:

$\mu=$ the overall mean; $S_{j}=$ the fixed effect of season of the year $(1=$ mild and $2=$ hot $) ; B_{i}=$ the fixed effect 
of breed type $\left(1=\right.$ crossbred and $2=$ Baladi) $; \mathrm{BS}_{\mathrm{ij}}=$ the interaction between the breed type and season of the year; $b=$ regression coefficient of $\mathrm{Y}$ on metabolic weight; $\overline{\mathrm{x}}=$ the arithmetic mean of metabolic weight; $\mathrm{e}_{\mathrm{ijk}}=$ random error.

Significance of the difference in the results was verified by Duncan's new multiple ranges test (Duncan, 1955).

\section{Results}

Effect of estrous cycle on experimental measurements:

\section{Rectal temperature (RT):}

Analysis of covariance for obtained data revealed highly significant variations in RT values due to season $(\mathrm{P}<0.0001)$ in all estrous cycle phases (Table 1). All values recorded were within the normal range of the goat breeds. Rectal Temperature was significantly lower in mild season compared with in hot season during estrous cycle. It recorded highest value $40.03 \pm 0.11{ }^{\circ} \mathrm{C}$ during diestrus phase and lowest value $39.69 \pm 0.14{ }^{\circ} \mathrm{C}$ during metestrus phase in hot season, however in goat does subjected to mild season it recorded highest value $39.01 \pm 0.21{ }^{\circ} \mathrm{C}$ during diestrus and lowest value $38.38 \pm 0.20{ }^{\circ} \mathrm{C}$ during estrus phases. Change rate was $+3.8 \%$, $+2.3 \%,+2.6 \%$ and $+3.7 \%$ during estrus, metestrus, diestrus and proestrus, respectively in hot season.

On the other hand, RT showed significant variation due to breed $(\mathrm{P}<0.05$ or $\mathrm{P}<0.01)$ during estrus phases, RT in Baladi goat does recorded highest value during proestrus phase $39.9 \pm 0.24{ }^{\circ} \mathrm{C}$ and lowest value during estrus phase $39.63 \pm 0.26{ }^{\circ} \mathrm{C}$. Similar trend of increase, but with lower rate was found in crossbred goat does, which recorded highest value during proestrus phase $39.59 \pm 0.21{ }^{\circ} \mathrm{C}$ and lowest value during estrus phase $38.6 \pm 0.24{ }^{\circ} \mathrm{C}$ with change rate $-2.6 \%,-2.7 \%,-1.6 \%$ and $-0.8 \%$ during estrus, metestrus, diestrus and proestrus, respectively in crossbred goat does (Table 1).

In respect of interaction between season and breed showed significant effect $(\mathrm{P}<0.01)$ in $\mathrm{RT}$ values during metestrus, also $(\mathrm{P}<0.05)$ during diestrus and insignificant variation during proestrus and estrus phases as shown in Table 1.

\section{Skin temperature (ST):}

Skin temperature was significantly lower $(\mathrm{P}<0.0001)$ in mild season than in hot season during estrous cycle. It recorded highest value $39.63 \pm 0.07{ }^{\circ} \mathrm{C}$ during proestrus phase and lowest value $39.46 \pm 0.11$
${ }^{\circ} \mathrm{C}$ during metestrus phase in hot season, however in goat does subjected to mild season it recorded highest value $38.19 \pm 0.11{ }^{\circ} \mathrm{C}$ during diestrus and lowest value $37.09 \pm 0.15{ }^{\circ} \mathrm{C}$ during metestrus phases. Change rate was $+5.2 \%,+6.4 \%,+3.5 \%$ and $+4.1 \%$ during estrus, metestrus, diestrus and proestrus, respectively in hot season as shown in Table 1.

On the other side, ST showed insignificant variation due to breed effect, ST in Baladi goat does recorded highest during proestrus phase $38.98 \pm 0.22{ }^{\circ} \mathrm{C}$ and lowest value during metestrus phase $38.19 \pm 0.38{ }^{\circ} \mathrm{C}$, however in crossbred goat does ST recorded highest value during diestrus phase $38.82 \pm 0.23{ }^{\circ} \mathrm{C}$ and lowest value during metestrus phase $38.36 \pm 0.37{ }^{\circ} \mathrm{C}$ with change rate $+0.4 \%,+0.4 \%,-0.2 \%$ and $-0.6 \%$ during estrus, metestrus, diestrus and proestrus, respectively in crossbred goat does (Table 1).

Interaction between season and breed showed insignificant variation in ST values during all phases of estrous cycle (Table 1).

\section{Respiration rate ( $R R)$ :}

Respiration rate was significantly lower $(\mathrm{P}<0.0001)$ in mild season than in hot season during estrous cycle. Respiration rate values increased in goat does subjected to hot season during diestrus and proestrus $26.75 \pm 0.51$ and $27.17 \pm 0.81 \quad$ breathes $/ \mathrm{min}$., respectively then decreased thereafter to reach its minimum value at metaestrus $(21.92 \pm 0.45$ breathes/min.). However, the rate of increase or decrease differed according to the estrous cycle phases. Similar trend of increase, but with lower rate was found in goat does subjected to mild season during diestrus and proestrus $(15.83 \pm 0.87$ and 17.75 \pm 0.79 breathes/min.), respectively then decreased thereafter to reach its minimum value at metaestrus $(15.67 \pm 1.12$ breathes/min.). Change rate was $+41.05 \%,+39.9 \%,+68.9 \%$ and $+53.1 \%$ during estrus, metestrus, diestrus and proestrus, respectively in hot season (Table 1).

Respiration rate showed insignificant variation due to breed effect, RR during estrous cycle was lower in crossbred, it recorded $(19 \pm 1.12,18 \pm 1.18,20.42 \pm 1.74$ and $22 \pm 1.92$ breathes/min.) compared with Baladi goat does $(20.58 \pm 1.79,19.58 \pm 1.32,22.16 \pm 1.81$ and $22.92 \pm 1.27$ breathes/min.) during estrus, metestrus, diestrus and proestrus, respectively. Change rate was $-7.7 \%,-8.1 \%,-7.9 \%$ and $-4.01 \%$ during estrus, metestrus, diestrus and proestrus, respectively in crossbred goat does (Table 1).

Interaction between season and breed showed insignificant variation in RR values during all phases of estrous cycle (Table 1). 
Table 1. Least square means $( \pm \mathrm{SE})$ for factors affecting rectal temperature, skin temperature $\left({ }^{\circ} \mathrm{C}\right)$ and respiration rate (breathes/min) in goat does during estrous cycle.

\begin{tabular}{|c|c|c|c|c|c|c|c|c|c|c|c|c|}
\hline \multirow{3}{*}{ Items } & \multicolumn{12}{|c|}{ Estrous cycle phases } \\
\hline & \multicolumn{3}{|l|}{ Estrus } & \multicolumn{3}{|l|}{ Metestrus } & \multicolumn{3}{|l|}{ Diestrus } & \multicolumn{3}{|l|}{ Proestrus } \\
\hline & RT & ST & $\mathbf{R R}$ & RT & ST & $\mathbf{R R}$ & RT & ST & $\mathbf{R R}$ & RT & ST & $\mathbf{R R}$ \\
\hline Season $(\mathbf{S})$ & $* * *$ & $* * *$ & $* * *$ & $* * *$ & $* * *$ & $* * *$ & $* * *$ & $* * *$ & $* * *$ & $* * *$ & $* * *$ & $* * *$ \\
\hline Mild & $\begin{array}{l}38.38^{\mathrm{B}} \\
\pm 0.20 \\
39.85^{\mathrm{A}} \pm 0.0 \\
8\end{array}$ & $\begin{array}{l}37.56^{\mathrm{B}} \\
\pm 0.21 \\
39.50^{\mathrm{A}} \\
\pm 0.15\end{array}$ & $\begin{array}{l}16.42^{\mathrm{B}} \\
\pm 0.77 \\
23.16^{\mathrm{A}} \\
\pm 1.38\end{array}$ & $38.81^{\mathrm{B}} \pm 0.25$ & $\begin{array}{l}37.09^{\mathrm{B}} \\
\pm 0.15 \\
39.46^{\mathrm{A}} \\
\pm 0.11\end{array}$ & $\begin{array}{l}15.67^{\mathrm{B}} \\
\pm 1.12 \\
21.92^{\mathrm{A}} \\
\pm 0.45\end{array}$ & $\begin{array}{l}39.01^{\mathrm{B}} \\
\pm 0.21 \\
40.03^{\mathrm{A}} \\
\pm 0.11\end{array}$ & $\begin{array}{l}38.19^{\mathrm{B}} \\
\pm 0.11 \\
39.53^{\mathrm{A}} \\
\pm 0.08\end{array}$ & $\begin{array}{l}15.83^{\mathrm{B}} \\
\pm 0.87 \\
26.75^{\mathrm{A}} \\
\pm 0.51\end{array}$ & $\begin{array}{l}38.54 \\
\mathrm{~B}_{ \pm 00.14} \\
39.95^{\mathrm{A}} \pm 0.1 \\
0\end{array}$ & $\begin{array}{l}38.08^{\mathrm{B}} \\
\pm 0.11 \\
39.63^{\mathrm{A}} \\
\pm 0.07\end{array}$ & $\begin{array}{l}17.75^{\mathrm{B}} \\
\pm 0.79 \\
27.17^{\mathrm{A}} \\
\pm 0.81\end{array}$ \\
\hline $\begin{array}{l}\text { Change(\% } \\
\text { ) }\end{array}$ & +3.8 & +5.2 & +41.05 & +2.3 & +6.4 & +39.9 & +2.6 & +3.5 & +68.9 & +3.7 & +4.1 & +53.1 \\
\hline P- value & 0.0001 & 0.0001 & 0.0003 & 0.0001 & 0.0001 & 0.0001 & 0.0001 & 0.0001 & 0.0001 & 0.0001 & 0.0001 & 0.0001 \\
\hline Breed (B) & $*$ & NS & NS & $* *$ & NS & NS & $*$ & NS & NS & $* *$ & NS & NS \\
\hline Baladi & $\begin{array}{l}39.63^{\mathrm{A}} \\
\pm 0.26\end{array}$ & $38.45 \pm 0.35$ & $20.58 \pm 1.79$ & $\begin{array}{l}39.79^{\mathrm{A}} \\
\pm 0.11\end{array}$ & $38.19 \pm 0.38$ & $19.58 \pm 1.32$ & $\begin{array}{l}39.84^{\mathrm{A}} \\
\pm 0.15\end{array}$ & $38.91 \pm 0.22$ & $22.16 \pm 1.81$ & $\begin{array}{l}39.90^{\mathrm{A}} \\
\pm 0.24\end{array}$ & $38.98 \pm 0.22$ & $22.92 \pm 1.27$ \\
\hline Crossbred & $38.6^{\mathrm{B}} \pm 0.24$ & $38.62 \pm 0.33$ & $19 \pm 1.12$ & $\begin{array}{l}38.71 \\
\pm 0.23\end{array}$ & $38.36 \pm 0.37$ & $18 \pm 1.18$ & $\begin{array}{l}39.20^{\mathrm{B}} \\
\pm 0.25\end{array}$ & $38.82 \pm 0.23$ & $20.42 \pm 1.74$ & $\begin{array}{l}39.59^{\mathrm{B}} \\
\pm 0.21\end{array}$ & $38.73 \pm 0.27$ & $22 \pm 1.92$ \\
\hline $\begin{array}{l}\text { Change(\% } \\
\text { ) }\end{array}$ & -2.6 & +0.4 & -7.7 & -2.7 & +0.4 & -8.1 & -1.6 & -0.2 & -7.9 & -0.8 & -0.6 & -4.01 \\
\hline P- value & 0.0145 & 0.7669 & 0.7596 & 0.0010 & 0.6821 & 0.1169 & 0.0125 & 0.9249 & 0.2389 & 0.0002 & 0.4209 & 0.6874 \\
\hline $\mathbf{S}^{*} \mathbf{T}$ & NS & NS & NS & $* *$ & NS & NS & $*$ & NS & NS & NS & NS & NS \\
\hline \multicolumn{13}{|l|}{ Mild } \\
\hline Baladi & $38.86 \pm 0.22$ & $37.35 \pm 0.23$ & $17.00 \pm 1.00$ & $39.58^{\mathrm{b}} \pm 0.06$ & $37.01 \pm 0.25$ & $16.83 \pm 1.99$ & $\begin{array}{l}39.45^{\mathrm{b}} \\
\pm 0.13\end{array}$ & $38.28 \pm 0.16$ & $16.83 \pm 1.58$ & $38.95 \pm 0.14$ & $38.30 \pm 0.11$ & $19.33 \pm 0.71$ \\
\hline Crossbred & $37.90 \pm 0.19$ & $37.78 \pm 0.34$ & $15.83 \pm 1.22$ & $38.05^{\mathrm{c}} \pm 0.20$ & $37.17 \pm 0.18$ & $14.50 \pm 1.02$ & $\begin{array}{l}38.58^{\mathrm{c}} \\
\pm 0.32\end{array}$ & $38.10 \pm 0.15$ & $14.83 \pm 0.65$ & $38.13 \pm 0.05$ & $37.87 \pm 0.16$ & $16.16 \pm 1.14$ \\
\hline $\begin{array}{l}\text { Change } \\
(\%)\end{array}$ & -2.5 & +1.2 & -6.9 & -3.9 & +0.4 & -13.8 & -2.2 & -0.5 & -11.9 & -2.1 & -1.1 & -16.4 \\
\hline \multicolumn{13}{|l|}{ Hot } \\
\hline Baladi & $40.40 \pm 0.11$ & $39.55 \pm 0.12$ & $25.33 \pm 1.87$ & $40.00^{\mathrm{a}} \pm 0.18$ & $39.37 \pm 0.18$ & $22.33 \pm 0.80$ & $\begin{array}{l}40.24^{\mathrm{a}} \\
\pm 0.15\end{array}$ & $39.53 \pm 0.17$ & $27.50 \pm 0.72$ & $40.23 \pm 0.10$ & $39.65 \pm 0.14$ & $27.83 \pm 1.14$ \\
\hline Crossbred & $39.31 \pm 0.14$ & $39.47 \pm 0.29$ & $21.00 \pm 1.71$ & $39.38^{\mathrm{b}} \pm 0.10$ & $39.55 \pm 0.13$ & $21.50 \pm 0.43$ & $\begin{array}{l}39.83^{\mathrm{b}} \\
\pm 0.11\end{array}$ & $39.53 \pm 0.03$ & $26.00 \pm 0.63$ & $39.68 \pm 0.07$ & $39.60 \pm 0.03$ & $26.50 \pm 1.20$ \\
\hline $\begin{array}{l}\text { Change } \\
(\%)\end{array}$ & -2.7 & -0.2 & -17.1 & -1.6 & +0.5 & -3.7 & -1.02 & 0 & -5.5 & -1.4 & -0.1 & -4.8 \\
\hline$P$ - value & 0.6675 & 0.3583 & 0.0611 & 0.0081 & 0.8250 & 0.4473 & 0.0353 & 0.5785 & 0.7767 & 0.1555 & 0.1474 & 0.0676 \\
\hline
\end{tabular}


Effect of pregnancy stages on experimental measurements:

\section{Rectal temperature (RT):}

Inspection the effect of season on RT it is noticeable that goat does recorded significant low $(\mathrm{P}<0.0001)$ RT under mild season in comparison with hot season during pregnancy stages (Table 2). Goat does recorded lowest $\mathrm{RT}$ value in late pregnancy stage with values about $36.65 \pm 0.26{ }^{\circ} \mathrm{C}$ and $39.35 \pm 0.15^{\circ} \mathrm{C}$ in mild season and hot season, respectively. However, it recorded highest value during early pregnancy with value $38.43 \pm 0.19{ }^{\circ} \mathrm{C}$ in mild season and $39.85 \pm 0.16{ }^{\circ} \mathrm{C}$ in hot season. Change rate was $+3.7 \%,+4.2 \%$ and $+7.4 \%$ during early, mid and late pregnancy, respectively in hot season.

From the other side, RT showed significant variation due to breed $(\mathrm{P}<0.05)$ during early and midpregnancy stages, also $(\mathrm{P}<0.0001)$ during late pregnancy stage, RT in Baladi goat does recorded highest value $\left(39.54 \pm 0.22{ }^{\circ} \mathrm{C}\right)$ during early and midpregnancy stages and lowest value during late pregnancy stage $38.62 \pm 0.36{ }^{\circ} \mathrm{C}$. Similar trend of increase, but with lower rate was found in crossbred goat does, which recorded highest value during early pregnancy $38.74 \pm 0.28{ }^{\circ} \mathrm{C}$ followed by midpregnancy $38.54 \pm 0.27{ }^{\circ} \mathrm{C}$ and lowest value during late pregnancy $37.37 \pm 0.47{ }^{\circ} \mathrm{C}$ with change rate $2.02 \%,-2.5 \%$ and $-3.2 \%$ during early, mid and late pregnancy stages, respectively in crossbred goat does (Table 2).

As presented in Table 2 interaction between season and breed showed significant effect $(\mathrm{P}<0.05)$ on $\mathrm{RT}$ values during late pregnancy stage, while insignificant variation during early and mid-pregnancy stages 2 .

\section{Skin temperature (ST):}

Skin temperature during pregnancy stages was lower in mild $(\mathrm{P}<0.0001)$ compared with hot season, it recorded $36.95 \pm 0.19,36.63 \pm 0.13$ and $35.96 \pm 0.22^{\circ} \mathrm{C}$ in mild season and $39.25 \pm 0.11,39.51 \pm 0.09$ and $38.89 \pm 0.16^{\circ} \mathrm{C}$ in hot season during early, mid and late pregnancy, respectively with change rate was $+6.2 \%,+7.9 \%$ and $+8.1 \%$ during early, mid and late pregnancy stages, respectively in hot season (Table 2 ).
On the other hand, ST showed insignificant variation due to breed effect, ST in Baladi goat does recorded highest during early pregnancy stage $37.99 \pm 0.44{ }^{\circ} \mathrm{C}$ and lowest value during late pregnancy stage $37.35 \pm 0.44$ ${ }^{\circ} \mathrm{C}$, similar trend was found in crossbred goat does ST recorded highest value during early pregnancy stage $38.21 \pm 0.29^{\circ} \mathrm{C}$ and lowest value during late pregnancy stage $37.50 \pm 0.52{ }^{\circ} \mathrm{C}$ with change rate $+0.6 \%,+0.7 \%$ and $+0.4 \%$ during early, mid and late pregnancy stages, respectively in crossbred goat does (Tabl4e 2).

Skin temperature values showed insignificant variation due to interaction between breed and season during all pregnancy stages, except early pregnancy stage showed significant effect $(\mathrm{P}<0.05)$ on $\mathrm{ST}$ values as shown in Table 2.

\section{Respiration rate ( $R R)$ :}

During pregnancy stages Baladi and crossbred goat does recorded significant low $(\mathrm{P}<0.0001) \mathrm{RR}$ under mild season during early pregnancy with value about $24.08 \pm 0.5$ breathes/min., also recorded significant high during late pregnancy with value about $26.08 \pm 0.58$ breathes/min., however the opposite recorded significant low at hot conditions during late pregnancy with value about 32.41 $\pm 0.77 \mathrm{breathes} / \mathrm{min}$. and high value during early pregnancy with value about $33.87 \pm 0.93$ breath $/ \mathrm{min}$. Change rate was $+40.7 \%$, $+29.3 \%$ and $+24.3 \%$ during early pregnancy, mid pregnancy and late pregnancy, respectively in hot season.

It can be seen from Table 2 that RR showed insignificant variation due to breed effect, RR during pregnancy period was lower in crossbred it recorded $28.33 \pm 1.49, \quad 28.50 \pm 1.37$ and $28.41 \pm 1.13$ breathes/min. compared with Baladi goat does with values $29.62 \pm 1.78,29.20 \pm 1.14$ and $30.08 \pm 1.16$ breathes/min. during early, mid and late pregnancy stages, respectively. Change rate was $-4.4 \%,-2.4 \%$ and $-5.6 \%$ during early, mid and late pregnancy stages respectively in crossbred goat does.

As shown in Table 2 RR values showed insignificant variation due to interaction between breed and season during all pregnancy stages. 
Table 2. Least square means $\left( \pm\right.$ SE) for factors affecting rectal temperature, skin temperature $\left({ }^{\circ} \mathrm{C}\right)$ and respiration rate (breathes/min) in goat does during pregnancy period.

\begin{tabular}{|c|c|c|c|c|c|c|c|c|c|}
\hline \multirow{3}{*}{ Items } & \multicolumn{9}{|c|}{ Pregnancy Period } \\
\hline & \multicolumn{3}{|c|}{ Early pregnancy } & \multicolumn{3}{|c|}{ Mid-pregnancy } & \multicolumn{3}{|c|}{ Late pregnancy } \\
\hline & RT & ST & $\mathbf{R R}$ & RT & ST & $\mathbf{R R}$ & RT & ST & $\mathbf{R R}$ \\
\hline Season $(S)$ & $* * *$ & $* * *$ & $* * *$ & $* * *$ & $* * *$ & $* * *$ & $* * *$ & $* * *$ & $* * *$ \\
\hline Mild & $38.43^{\mathrm{B}} \pm 0.19$ & $36.95^{\mathrm{B}} \pm 0.19$ & $24.08^{\mathrm{B}} \pm 0.50$ & $38.23^{\mathrm{B}} \pm 0.19$ & $36.63^{\mathrm{B}} \pm 0.13$ & $25.16^{\mathrm{B}} \pm 0.49$ & $36.65^{\mathrm{B}} \pm 0.26$ & $35.96^{\mathrm{B}} \pm 0.22$ & $26.08^{\mathrm{B}} \pm 0.58$ \\
\hline Hot & $39.85^{\mathrm{A}} \pm 0.16$ & $39.25^{\mathrm{A}} \pm 0.11$ & $33.87^{\mathrm{A}} \pm 0.93$ & $39.85^{\mathrm{A}} \pm 0.16$ & $39.51^{\mathrm{A}} \pm 0.09$ & $32.54^{\mathrm{A}} \pm 0.69$ & $39.35^{\mathrm{A}} \pm 0.15$ & $38.89^{\mathrm{A}} \pm 0.16$ & $32.41^{\mathrm{A}} \pm 0.77$ \\
\hline Change(\%) & +3.7 & +6.2 & +40.7 & +4.2 & +7.9 & +29.3 & +7.4 & +8.1 & +24.3 \\
\hline$P$ - value & 0.0001 & 0.0001 & 0.0001 & 0.0001 & 0.0001 & 0.005 & 0.0001 & 0.0001 & 0.0001 \\
\hline Breed (B) & $*$ & NS & NS & $*$ & NS & NS & $* * *$ & NS & NS \\
\hline Baladi & $39.54^{\mathrm{A}} \pm 0.22$ & $37.99 \pm 0.44$ & $29.62 \pm 1.78$ & $39.54^{\mathrm{A}} \pm 0.22$ & $37.95 \pm 0.44$ & $29.20 \pm 1.14$ & $38.62^{\mathrm{A}} \pm 0.36$ & $37.35 \pm 0.44$ & $30.08 \pm 1.16$ \\
\hline Crossbred & $38.74^{\mathrm{B}} \pm 0.28$ & $38.21 \pm 0.29$ & $28.33 \pm 1.49$ & $38.54^{\mathrm{B}} \pm 0.27$ & $38.20 \pm 0.45$ & $28.50 \pm 1.37$ & $37.37^{\mathrm{B}} \pm 0.47$ & $37.50 \pm 0.52$ & $28.41 \pm 1.13$ \\
\hline Change $(\%)$ & -2.02 & +0.6 & -4.4 & -2.5 & +0.7 & -2.4 & -3.2 & +0.4 & -5.6 \\
\hline P- value & 0.0166 & 0.9375 & 0.1648 & 0.0207 & 0.1674 & 0.9421 & 0.0001 & 0.6738 & 0.1359 \\
\hline $\mathbf{S} * \mathbf{T}$ & NS & $*$ & NS & NS & NS & NS & $*$ & NS & NS \\
\hline \multicolumn{10}{|l|}{ Mild } \\
\hline Baladi & $39.00 \pm 0.16$ & $36.58^{\mathrm{c}} \pm 0.24$ & $24.25 \pm 0.87$ & $39.00 \pm 0.16$ & $36.52 \pm 0.15$ & $25.83 \pm 0.54$ & $37.43^{\mathrm{c}} \pm 0.07$ & $36.08 \pm 0.41$ & $26.66 \pm 0.80$ \\
\hline Crossbred & $37.87 \pm 0.15$ & $37.31^{\mathrm{b}} \pm 0.19$ & $23.91 \pm 0.58$ & $37.67 \pm 0.15$ & $36.75 \pm 0.20$ & $24.50 \pm 0.79$ & $35.87^{\mathrm{d}} \pm 0.21$ & $35.85 \pm 0.22$ & $25.50 \pm 0.85$ \\
\hline Change( $\%)$ & -2.9 & +1.9 & -1.4 & -3.4 & +0.6 & -5.1 & -4.2 & -0.6 & -4.4 \\
\hline \multicolumn{10}{|l|}{ Hot } \\
\hline Baladi & $40.09 \pm 0.27$ & $39.40^{\mathrm{a}} \pm 0.14$ & $35.00 \pm 1.29$ & $40.09 \pm 0.27$ & $39.38 \pm 0.13$ & $32.58 \pm 0.93$ & $39.81^{\mathrm{a}} \pm 0.05$ & $38.61 \pm 0.17$ & $33.50 \pm 0.81$ \\
\hline Crossbred & $39.61 \pm 0.11$ & $39.40^{\mathrm{a}} \pm 0.03$ & $32.75 \pm 1.27$ & $39.41 \pm 0.11$ & $39.65 \pm 0.12$ & $32.50 \pm 1.11$ & $38.88^{\mathrm{b}} \pm 0.12$ & $39.16 \pm 0.24$ & $31.33 \pm 1.23$ \\
\hline Change(\%) & -1.2 & -0.8 & -6.4 & -1.7 & +0.7 & -0.2 & -2.3 & +1.4 & -6.5 \\
\hline P- value & 0.0956 & 0.0182 & 0.4523 & 0.1884 & 0.9728 & 0.5366 & 0.0179 & 0.1927 & 0.6795 \\
\hline
\end{tabular}

Least square means with different letters (A and B or a, b, c and d) in the same column are significantly different at $(\mathrm{P}<0.05)$.

$*=\mathrm{P}<0.05, * * * \mathrm{P}<0.0001$ and $\mathrm{NS}=$ not significant 
Effect of postpartum period on experimental measurement:

\section{Rectal temperature (RT):}

Rectal temperature recorded significantly higher values $(\mathrm{P}<0.01)$ in hot season compared with mild season during day of parturition and 15 days postpartum, while $(\mathrm{P}<0.0001)$ during 30 days postpartum (Table 3). In hot season goat does recorded highest RT value during 15 days postpartum $\left(39.5 \pm 0.08{ }^{\circ} \mathrm{C}\right)$ and 30 days postpartum $(39.5 \pm 0.07$ ${ }^{\circ} \mathrm{C}$ ), however it recorded lowest value during day of parturition $\left(39.27 \pm 0.13{ }^{\circ} \mathrm{C}\right)$, similar trend but with lower rate was found in mild season goat does recorded highest value during 15 days postpartum $\left(38.75 \pm 0.12^{\circ} \mathrm{C}\right)$ and 30 days postpartum $(38.38 \pm 0.10$ ${ }^{\circ} \mathrm{C}$ ), however it recorded lowest value during day of parturition $\left(38.66 \pm 0.21{ }^{\circ} \mathrm{C}\right)$. Change rate was $+1.6 \%$, $+1.9 \%$, $+2.9 \%$ during day of parturition, 15 days postpartum and 30 days postpartum, respectively in hot season (Table 3).

On the other side, RT showed significant variation due to breed $(\mathrm{P}<0.05)$ during day of parturition and 15 days postpartum stages, while insignificant effect during 30 days postpartum stage, RT in Baladi goat does recorded highest value $\left(39.10 \pm 0.18{ }^{\circ} \mathrm{C}\right)$ during day of parturition stage and lowest value during 30 days postpartum stage $38.85 \pm 0.19{ }^{\circ} \mathrm{C}$, while in crossbred goat does recorded highest value during 15 days postpartum $39.08 \pm 0.13{ }^{\circ} \mathrm{C}$ and lowest value during day of parturition $38.84 \pm 0.21{ }^{\circ} \mathrm{C}$ with change rate $-0.7 \%,-0.2 \%$ and $+0.5 \%$ during day of parturition, 15 days postpartum and 30 days postpartum stages, respectively in crossbred goat does (Table 3).

Interaction between season and breed showed insignificant effect in RT values during all postpartum period (Table 3 ).

\section{Skin temperature (ST):}

Skin temperature almost significantly higher under hot season $\left({ }^{\circ} \mathrm{C}\right)$ along the postpartum periods in comparison with mild season $(\mathrm{P}<0.01)$ during day of parturition and $(\mathrm{P}<0.0001)$ during 15 days postpartum and 30 days postpartum. Under hot season goat does recorded high ST at 15 days postpartum $(39.5 \pm 0.08$ $\left.{ }^{\circ} \mathrm{C}\right)$ and low ST at day of parturition $(38.63 \pm 0.14$ $\left.{ }^{\circ} \mathrm{C}\right)$. However, under mild season goat does recorded high ST at day of parturition $\left(37.75 \pm 0.21^{\circ} \mathrm{C}\right)$ and low ST at 30 days postpartum $\left(37.37 \pm 0.18{ }^{\circ} \mathrm{C}\right)$. Change rate was $+2.3 \%,+5.2 \%$ and $3.8 \%$ during day of parturition, 15 days postpartum and 30 days postpartum, respectively in hot season.

On the other hand, ST showed insignificant variation due to breed effect during day of parturition and 15 days postpartum, while significantly affected $(\mathrm{P}<0.01)$ during 30 days postpartum. Skin temperature in Baladi goat does significantly higher $(\mathrm{P}<0.01)$ in comparison with crossbred goat does during 30 days postpartum, in Baladi goat does recorded highest value during 30 days postpartum stage $\left(38.18 \pm 0.25^{\circ} \mathrm{C}\right)$ and lowest value during day of parturition stage $\left(38.06 \pm 0.24{ }^{\circ} \mathrm{C}\right)$, in crossbred goat does ST recorded highest value during day of parturition stage $\left(38.31 \pm 0.21{ }^{\circ} \mathrm{C}\right)$ and lowest value during 30 days postpartum stage $\left(38.00 \pm 0.27{ }^{\circ} \mathrm{C}\right)$ with change rate $+0.7 \%,+0.2 \%$ and $-0.5 \%$ during day of parturition, 15 days postpartum and 30 days postpartum stages, respectively in crossbred goat does as shown in Table 3.

Skin temperature values showed insignificant variation due to interaction between breed and season during all postpartum stages, except 15 days postpartum $(\mathrm{P}<0.05)$ as shown in Table 3.

\section{Respiration rate ( $R R)$ :}

$\mathrm{RR}$ recorded significantly higher values in hot season than mild season during all postpartum periods $(\mathrm{P}<0.05)$ during day of parturition and 15days postpartum, while $(\mathrm{P}<0.0001)$ during 30 days postpartum, it recorded highest value during 15 days postpartum $(27.75 \pm 0.65$ breathes $/ \mathrm{min})$ and lowest value during 30 days postpartum $(24.91 \pm 0.47$ breathes/min) in mild season, also recorded highest value during 30 days postpartum (29.58 \pm 0.69 breathes/min) and lowest value during 15 days postpartum $(29.16 \pm 0.79$ breathes $/ \mathrm{min})$ in hot season with change rate $+9.6 \%,+5.1 \%$ and $+18.7 \%$ during day of parturition, 15 days postpartum and 30 days postpartum, respectively in hot season (Table 3 ).

Respiration rate showed significant variation due to breed effect in all postpartum stages, $(\mathrm{P}<0.01)$ during 15 days postpartum and 30 days postpartum and $(\mathrm{P}<0.05)$ during day of parturition, RR during postpartum period was lower in crossbred it recorded $26.41 \pm 0.86, \quad 27.00 \pm 0.54$ and $26.08 \pm 0.88$ breathes/min. compared with Baladi goat does $(29.83 \pm 0.93, \quad 29.91 \pm 0.69$ and $28.41 \pm 0.81$ breathes/min.) during day of parturition, 15 days postpartum and 30 days postpartum stages, respectively. Change rate was $-11.5 \%,-9.7 \%$ and $8.2 \%$ during during day of parturition, 15 days postpartum and 30 days postpartum stages, respectively in crossbred goat does (Table 3 ).

Respiration rate values showed insignificant variation due to interaction between breed and season during all postpartum stages, except 15 days postpartum $(\mathrm{P}<0.05)$ as shown in Table 3 . 
Table 3. Least square means $( \pm \mathrm{SE})$ for factors affecting rectal temperature, skin temperature $\left({ }^{\circ} \mathrm{C}\right)$ and respiration rate (breathes/min) in goat does during postpartum period.

\begin{tabular}{|c|c|c|c|c|c|c|c|c|c|}
\hline \multirow{3}{*}{ Items } & \multicolumn{9}{|c|}{ Postpartum period } \\
\hline & \multicolumn{3}{|c|}{ Day of parturition } & \multicolumn{3}{|c|}{ 15days Postpartum } & \multicolumn{3}{|c|}{ 30days Postpartum } \\
\hline & RT & ST & $\mathbf{R R}$ & RT & ST & $\mathbf{R R}$ & RT & ST & $\mathbf{R R}$ \\
\hline Season $(S)$ & $* *$ & $* *$ & $*$ & $* *$ & $* * *$ & $*$ & $* * *$ & $* * *$ & $* * *$ \\
\hline Mild & $38.66^{\mathrm{B}} \pm 0.21$ & $37.75^{\mathrm{B}} \pm 0.21$ & $26.83^{\mathrm{B}} \pm 1.06$ & $38.75^{\mathrm{B}} \pm 0.12$ & $37.56^{\mathrm{B}} \pm 0.19$ & $27.75^{\mathrm{B}} \pm 0.65$ & $38.38^{\mathrm{B}} \pm 0.10$ & $37.37^{\mathrm{B}} \pm 0.18$ & $24.91^{\mathrm{B}} \pm 0.47$ \\
\hline Hot & $39.27^{\mathrm{A}} \pm 0.13$ & $38.63^{\mathrm{A}} \pm 0.14$ & $29.41^{\mathrm{A}} \pm 0.83$ & $39.50^{\mathrm{A}} \pm 0.08$ & $39.50^{\mathrm{A}} \pm 0.08$ & $29.16^{\mathrm{A}} \pm 0.79$ & $39.50^{\mathrm{A}} \pm 0.07$ & $38.80^{\mathrm{A}} \pm 0.09$ & $29.58^{\mathrm{A}} \pm 0.69$ \\
\hline Change $(\%)$ & +1.6 & +2.3 & +9.6 & +1.9 & +5.2 & +5.1 & +2.9 & +3.8 & +18.7 \\
\hline$P$ - value & 0.0026 & 0.0038 & 0.0360 & 0.0012 & 0.0001 & 0.0494 & 0.0001 & 0.0001 & 0.0001 \\
\hline Breed (B) & $*$ & NS & $*$ & $*$ & NS & $* *$ & NS & $* *$ & $* *$ \\
\hline Baladi & $39.10^{\mathrm{A}} \pm 0.18$ & $38.06 \pm 0.24$ & $29.83^{\mathrm{A}} \pm 0.93$ & $39.17^{\mathrm{A}} \pm 0.17$ & $38.13 \pm 0.22$ & $29.91^{\mathrm{A}} \pm 0.69$ & $38.85 \pm 0.19$ & $38.18^{\mathrm{A}} \pm 0.25$ & $28.41^{\mathrm{A}} \pm 0.81$ \\
\hline Crossbred & $38.84^{\mathrm{B}} \pm 0.21$ & $38.31 \pm 0.21$ & $26.41^{\mathrm{B}} \pm 0.86$ & $39.08^{\mathrm{B}} \pm 0.13$ & $38.20 \pm 0.26$ & $27.00^{\mathrm{B}} \pm 0.54$ & $39.03 \pm 0.19$ & $38.00^{\mathrm{B}} \pm 0.27$ & $26.08^{\mathrm{B}} \pm 0.88$ \\
\hline Change(\%) & -0.7 & +0.7 & -11.5 & -0.2 & +0.2 & -9.7 & +0.5 & -0.5 & -8.2 \\
\hline$P$ - value & 0.0197 & 0.9712 & 0.0403 & 0.0415 & 0.2740 & 0.0011 & 0.4534 & 0.0039 & 0.0034 \\
\hline $\mathbf{S}^{*} \mathbf{T}$ & NS & NS & NS & NS & $*$ & $*$ & NS & NS & NS \\
\hline \multicolumn{10}{|l|}{ Mild } \\
\hline Baladi & $37.73 \pm 0.19$ & $38.06 \pm 0.24$ & $27.83 \pm 1.35$ & $38.90 \pm 0.14$ & $37.36^{\mathrm{b}} \pm 0.07$ & $28.33^{\mathrm{b}} \pm 0.99$ & $38.31 \pm 0.16$ & $37.20 \pm 0.14$ & $26.00 \pm 0.52$ \\
\hline Crossbred & $37.76 \pm 0.40$ & $38.31 \pm 0.21$ & $25.83 \pm 1.66$ & $38.61 \pm 0.18$ & $37.76^{\mathrm{b}} \pm 0.39$ & $27.16^{\mathrm{b}} \pm 0.87$ & $38.45 \pm 0.15$ & $37.55 \pm 0.33$ & $23.83 \pm 0.48$ \\
\hline Change $(\%)$ & -0.3 & +0.08 & -7.2 & -0.7 & +1.1 & -4.1 & +0.4 & +0.9 & -8.3 \\
\hline \multicolumn{10}{|l|}{ Hot } \\
\hline Baladi & $39.48 \pm 0.09$ & $38.36 \pm 0.21$ & $31.83 \pm 0.60$ & $39.55 \pm 0.13$ & $38.50^{\mathrm{a}} \pm 0.11$ & $31.50^{\mathrm{a}} \pm 0.34$ & $39.40 \pm 0.10$ & $38.81 \pm 0.10$ & $30.83 \pm 0.45$ \\
\hline Crossbred & $39.06 \pm 0.22$ & $38.90 \pm 0.10$ & $27.00 \pm 0.58$ & $39.45 \pm 0.11$ & $39.00^{\mathrm{a}} \pm 0.16$ & $26.83^{\mathrm{b}} \pm 0.70$ & $39.61 \pm 0.07$ & $38.80 \pm 0.18$ & $28.33 \pm 1.09$ \\
\hline Change $(\%)$ & -1.1 & +1.4 & -15.2 & -0.3 & +1.3 & -14.8 & +0.5 & -0.03 & -8.1 \\
\hline P-value & 0.7367 & 0.2482 & 0.2828 & 0.0846 & 0.0273 & 0.0336 & 0.7488 & 0.1687 & 0.6867 \\
\hline
\end{tabular}

Least square means with different letters ( $\mathrm{A}$ and $\mathrm{B}$ or $\mathrm{a}, \mathrm{b})$ in the same column are significantly different at $(\mathrm{P}<0.05)$.

$*=\mathrm{P}<0.05, * *=\mathrm{P}<0.01, * * * \mathrm{P}<0.0001$ and $\mathrm{NS}=$ not significant 


\section{Discussion}

The present results revealed that RT was significant increased during hot than mild season throughout estrous cycle, pregnancy stages and postpartum periods. These increases in RT under hot summer conditions indicated that animals were subjected to heat stress (Alamer and Al-Hozab, 2004; AlHaidary et al., 2012; Minka and Ayo, 2012; AlSamawi et al., 2014). Animals maintain the body temperature thermal equilibrium congeners dispersion of excess heat from their bodies, when exposed to high temperatures. This is accompanied by many biological functions, and that an increase in the rate of breathing or panting is the most obvious reaction. Under these circumstances, the animal body temperature expressed in increased body temperature when the body fails to maintain thermal equilibrium (Marai et al., 2007). Furthermore, Fahmy (1994) and Marai et al., (1997) reported that heat stress (HS), increased RT in goats.

The significant increase in RT (increase in heat storage) during hot season may be due to insufficient heat loss mechanisms as suggested by LU (1989) or it may be a thermal ability which decreases heat gain from the surrounding atmosphere by convection, conduction and radiation, showing adaptation of the animal as suggested by MacFarlane (1968) on camel. In the present study it may be due to insufficient heat loss because heat production increased with the increase in body temperature. Dahlanuddin and Thwaites (1993) reported that the limits of heat tolerance for goats is between 35 to 40 ${ }^{\circ} \mathrm{C}$ and the thermoneutral zone was suggested to be 25 to $30{ }^{\circ} \mathrm{C}$ above which rectal temperature may rise due to insufficient heat loss mechanisms. Also, Ominski et al., (2002) found that the relatively high environmental temperature increased RT. Furthermore, McDowell et al., (1976) reported that a rise of $1^{\circ} \mathrm{C}$ or less in $\mathrm{RT}$ is enough to reduce performance in most livestock species, which makes rectum temperature (body temperature) is a sensitive indicator to ambient temperature in dairy cows. On the other hand presented results disagree with findings of Itoh et al., (1998) who reported that the RT was not affected by heat exposure in nonlactating dairy cows. Obtained results agree with Ogebe et al. (1996) who informed that season significantly affect rectal temperature of purebred and crossbred goats.

Generally, during estrous cycle, pregnancy and postpartum period ST obviously and significantly decreased during mild season compared with hot season. These results are in agreement with Khalifa(1982); El-Sherbiny et al., (1983); ElShafie (1997); Abd El- Ghany (1997) and Abd ElKhalek (1997). Shalaby (1985) reported that seasonal change in ST of sheep was maximum values during summer and minimum during winter. The relation between ST and (AT) was explained by Folk (1974) as a function of blood vessels constriction during cold weather (vasoconstriction) to reduce blood flow and heat loss from the skin, while vasodilation at high temperature will result in increased blood flow and heat loss from the body. In addition, El-Sherbiny et al., (1983) found that increasing air temperature from $10^{\circ} \mathrm{C}$ to $40^{\circ} \mathrm{C}$ increased skin temperature significantly. Moreover, Montsma et al., (1985) stated that ST was more affected by ambient temperature than rectal temperature. Range of ST (37.0 to $39.4^{\circ} \mathrm{C}$ ) was wider than that of rectal temperature $\left(38.9\right.$ to $\left.39.9^{\circ} \mathrm{C}\right)$ when AT ranged from $20-35^{\circ} \mathrm{C}$. Furthermore, significant increase in ST during summer was related to vasodilatation of skin capillary bed and consequently increases the blood flow to the skin surface to facilitate heat dissipation (McManus et al., 2009). Significant decrease of ST during winter was related to compensatory blood redistribution from the peripheral back to the visceral regions of the body (Al-Tamimi, 2007).

The current results are in agreement with the finding of Okourwa et al., (2013) who reported that, respiratory rate is practical and reliable measure of heat load and stated that respiratory rate above 12 to 20 breath/minute in sheep and goats is an indicator of heat stress. As well as, Minka and Ayo, (2012) stated that increase of respiratory frequency following heat stress (HS) had been previously reported in livestock, including goats. Also, Alamer and Al-Hozab, (2004) demonstrated that RR was used as an indicator of HS, and used to estimate the negative effects of the environmental temperature. The increase of RR during summer season in the present study indicate HS and animal panting was one of the mechanisms used to dissipate excess heat through evaporation (Marai et al., 2007 and AlHaidary et al., 2012).

Rectal temperature results for the effect of breed illustrated that crossbred goat does were less affected by climatic condition compared with purebred goat does. It was found that rectal temperature of Baladi goat does was smaller high than those of crossbred; these results disagree with Muller and Botha (1998) who reported that crossbred Sindhi-Jersey cows showed a smaller increase in rectal temperature in comparison with pure Jersey cows under similar climatic conditions. On the other hand, obtained results agree with findings of Pastsart et al., (2006) and Molee et al., (2011) who found that Holstein crossed with local breeds in the tropics and subtropics perform better than the purebred Holstein and were also resistant to heat stress.

Skin temperature results showed no significantly difference between crossbred and purebred goat does during hot season. This result goes in agreement with Tarek, (2002) who reported that no significant differences were observed in skin temperature between different breeds during summer. On the other side, results obtained for the breed effect on RR indicated that insignificant variation between crossbred and Baladi goat does, while RR of Baladi 
goat does was slightly higher compared with those of crossbred, this results disagree with Olson et al., (2002) who reported that (Holstein $x$ Senepol) crossbred cattle had fewer breaths per minute ( $\mathrm{P}<$ 0.05) than purebred (Holstein cattle). While, Mahmut et al., (2006) informed that RR was significantly different among breeds and RR in purebred was smaller increase compared with crossbred goats.

In conclusion, this study revealed that crossed zaraibi with Baladi goat does perform better and were more adapted to the subtropical conditions of Egypt in comparison with the purebred Baladi goat does, it showed decrease in thermoregulatory responses especially rectal temperature during some stages of estrous, pregnancy and postpartum periods in particullary under hot season.

\section{References}

Abd El-Ghany, W.H. (1997). The role of wool coat of barki sheep in adaptation under semi-arid conditions. Ph.D.Thesis, Fac. Agric., Al-Azhar Univ., Cairo, Egypt.

Abd El-Khalek, T.M.M. (1997). Adaptability of goats under Egyptian environmental conditions. M.Sc. Thesis, Fac. Agric., Al-Azhar Univ., Cairo, Egypt.

Alam, M. M., Hashem, M. A., Rahman, M. M., Hossain, M. M., Haque, M. R., Sobhan, Z. and Islam, M. S. (2011): Effect of heat stress on behavior, physiological and blood parameters of goat. Progress. Agric. 22(1 \& 2): $37-45$.

Alamer, M. and Al-Hozab, A. (2004). Effect of water deprivation and season on feed intake, body weight and thermoregulation in Awassi and Najdi sheep breeds in Saudi Arabia. J. Arid. Environ. 59: 71-84.

Al-Haidary, A., Aljumaah, R.S., Alshaikh, M.A., Abdoun, K.A., Samara, E.M., Okab, A.B. and Alfuraiji, M.M., (2012). Thermoregulatory and physiological responses of Najdi sheep exposed to environmental heat load prevailing in Saudi Arabia. Pak. Vet. J. 32: 515-519.

Al-Samawi, K.A., Al-Hassan, M.J. and Swelum, A.A. (2014). Thermoregulation of female aardi goats exposed to environmental heat stress in Saudi arabia. Indian J. Anim. Res., 48(4):344-349.

Al-Tamimi, H.J. (2007). Thermoregulatory response of goat kids subjected to heat stress. Small Rumin. Res. 71: 280-285.

Atta, M.A.A. (2014). Estimating the adaptability of bovine calves to hot climatic conditions. Ph.D. Thesis, Fac. Agric., Zagazig Univ., Egypt.

Dahlanuddin, T.H. and Thwaites, C.J. (1993). Feed-water intake relations in goats of high ambient temperature. J. Anim. Physiol. and Anim. Nutr., 69:169.
Devendra, C. (1987): Goats. Ed. Johnson, H. P. In: Bioclimatology and the Adaptation of Livestock. Elsevier Publication Holland. pp. 16-77.

Duncan, D.B. (1955): Multiple range and multiple F-test. Biometrics, 11:1-42.

El-Shafie, M.H. (1997). Reflection of environmental adaptation on reproductive performance in indigenous and exogenous goats. M.Sc. Thesis, Fac. Agric., Cairo Univ., Giza, Egypt.

El-Sherbiny, A.A., Yousef, M.K., Salem, M.H., Khalifa, H.H., Abd El-Bary, H.T. and Khalil, M.H. (1983). Thermoregulatory responses of a desert and non-desert goat breed. Al-Azhar J. Agric. Res. Bull., 89.

Fahmy, S. (1994): Effect of crossing Romanov with Rahmani sheep on some physiological and productive performance. M. Sc. thesis. Fac. Agric. Al-Azhar Univ., Cairo, Egypt.

Folk, G.E. (1974). Textbook of Environmental physiology. $2^{\text {nd }}$ ED.Lea and Febiger, Philadelphia, USA.

Hansen, J. P. (2009): Effects of heat stress on mammalian reproduction. Phil.Trans.R. Soc. B., 364: 3341-3350.

Itoh, F.Y., Obara, M.T., Rose and Fuse, H. (1998). Heat influences on plasma insulin and glucagons in response to secretogogues in non- lactating dairy cows. Domest. Anim. Endocrin. 15(6): 499510.

Jordan, E. R. (2003): Effects of heat stress on reproduction. J. Dairy Sci. 86: E. Suppl.: E104E114.

Khalifa, H.H. (1982). Wool coat and thermoregulation in sheep under Egyptian conditions. Ph.D. Thesis, Fac. Agric., Al-Azhar Univ., Cairo, Egypt.

Khalifa, H. H.; El-Sherbiny, A. A. and AbdelKhalik, T. M. M., (2000): Effect of exposure to solar radiation on some adaptive physiological mechanisms of Egyptian goats. Proc. Conf. Anim. Prod. In the 12st Century, Sakha, 18-20 April 2000, pp. 297-305.

Lu, C.D. (1989). Effects of heat stress on goat production.Small Rumin. Res., 2:151.

Macfarlane, W. V. (1968). Adaptation of Ruminants to tropics and desert. Chapter, 12. In : Adaptation of Domestic Animals. Hafez, E.S.E. (ed.) Lea and Febiger, Philadelphia, USA, p. 180.

Mahmut K., Osman B., Sabri G. and Ayhan S. (2006). A study on comparison of some physiological adaptation parameters of different goat genotypes under the eastern mediterranean climatical condition. Hayvansal Üretim 47(1): 1620.

Marai, I.F.M., Bahgat, L.B., Shalaby, T.H. and Abdel-Hafez, M.A. (2000). Fattening performance, some behavioural traits and physiological reactions of male lambs fed concentrates mixture alone with or without 
natural clay, under hot summer of Egypt. Ann. Arid Zone, 39 (4): 449-460.

Marai, I. F. M., Daader, A. M., Abdel-Samee, A. M. and Ibrahim, H. (1997): Winter and summer effects and their ameleoration on lactating Friesian and Holstein cows maintained under Egyptian conditions. In: Proceedings of International Conference on Animal, Poultry, Rabbits and Fish Production and Health, Cairo, Egypt.

Marai, I.F.M.; El-Darawany, A.A.; Fadiel, A. and Abdel-Hafez, M.A.M. (2007). Physiological traits as affected by heat stress in sheep: a review. Small Rumin. Res. 71: 1-12.

Marai, I.F.M.; Habeeb, A.A.M. and Gad, A.E., (2002). Reproductive traits of female rabbits as affected by heat stress and light regime, under sub-tropical conditions of Egypt. J. Anim. Sci. $75,451-458$.

McDowell, R.E., Hooven, N.W. and Camoens, J.K. (1976). Effects of climate on performance of Holsteins in first lactation. J. Dairy Sci, 59, 965973.

McManus, C., Paludo, G.R., Louvandini, H., Gugel, R., Sasaki, L.C.B. and Paiva, S.R., (2009). Heat tolerance in Brazilian sheep: Physiological and blood parameters. Trop. Anim. Health Prod. 41: 95-101.

Minka, N.S., and Ayo, J.O. (2012). Assessment of thermal load on transported goats administered with ascorbic acid during the hot-dry conditions. Int. J. Biometeorol. 56:333-41.

Molee, A.; Bundasak, B.; Petladda, K. and Plern, M. (2011). Suitable percentage of Holstein incrossbred dairy cattle in climate changes ituation. J. Anim. Vet. Advnced, 10 (7): 828-831.

Montsma, G.; Luiting, P.; Verstegen, M.W.A.; Vander, Hel, W.; Hofs, P. and Zijlker, J.W. (1985). Effect of high ambient temperature on the metabolism of West African Dwarf goats .Inter. J. Biometeor., 29:23.

Muller, C.J.C., Botha J.A. (1998). The comparative performance of primiparous Holstein Friesland and Jersey cows on complete diets during summer in a temperate climate. S. Afr. J. Anim. Sci.; 28:161-166.

NRC. (2007). Nutrient Requirements of Small Ruminants: Sheep, Goats, Cervids, and New World Camelids. Natl. Acad. Press, Washington, DC.

Ogebe, P.O., Ogunmodede, B.K. and Mc Dowell, L.R. (1996). Behavioral and physiological responses of Nigerian Dwarf goats to seasonal changes of the humid tropics. Small Rumin. Res.; 22 (3): 213-217.

Okoruwa, M.I., Adewumi, M.K. and Igene, F.U. (2013). Thermophysiological responses of West African dwarf (WAD) bucks fed Pennisetum purpureum and unripe plantain peels. Nig. J. Anim Sci. 15: 168-178.
Olson,T.A. (2011). Crossbreeding programs for beef cattle in Florida publication. University of Florida, IFAS Extension, BUL, 326.

Olson, T.A.; Avila-Chytil, M.; Chase, C.C.Jr.; Hansen, P.J. and Coleman, S.W. (2002). Impact of hair coat differences on rectal temperature, skin temperature, and respiration rate of Holstein $X$ Senepol crosses in Florida. Senepol Symposium, St. Croix, USVI November 8-10.

Ominski, K.H., Kennedy, A.D., Wittenberg, K.M. and Moshtaghi Nia, S.A. (2002). Physiological and production responses to feeding scgedule in lactating dairy cows exposed to short-term, moderate heat stress. J.Dairy Sci. 85, 730-737.

Otoikhian, C.S.O., Orheruata, J.A., Imasuen, J.A. and Akporhuarho, O.P. (2009): Physiological response of local (West African Dwarf) and adapted switndzerla (White Bornu) goat breed to varied climatic conditions in South- South Nigeria. Afr. J. General Agric.; 5:1-6.

Pastsart, U.; Piyopummintr, A.; Kanjanapruthipong, J. and Siripholvat, V. (2006). Heat shock protein 90 (Hsp90) gene polymorphism associated with heat tolerance traits in crossbred dairy cattle and Thainative cattle. Journal of Agriculture Science 37: 393398.

Phulia, S.K., Upadhyay, R.C., Jindal, S.K. and Misra, R.P. (2010): Alteration in surface body temperature and physical responses in Sirohi goats during day time in summer season. Ind. J. Anim. Sci. 80: 340-342.

SAS (1991). Statistical Analysis System, SAS User's Guide Statistics, SAS Institute Inc., Editions Cary NC.

Shalaby, T. H. (1985). Performance and adaptation of local sheep to varied environmental and managerial conditions. Ph.D. Thesis, Fac. Agric., Cairo Univ., Giza, Egypt.

Sharma, S., Ramesh, K., Hyder, I., Uniyal, S. and Yadav, V.P. (2013): Effect of melatonin administration on thyroid hormones, cortisol and expression profile of heat shock proteins in goats (Capra hircus) exposed to heat stress. Small Ruminant Res., 112: 216-223.

Shebaita, M. K. and El-Banna, I. M. (1982): Heat load and heat dissipation in sheep and goats under environmental heat stress. Pp. 459-469 in Proc. 6th Int. Conf. Anim. Poult. Prod., Univ. Zagazig, Egypt.

Slee, J. (1966): Variation in the responses of shorn sheep to cold exposure. Anim. Prod. 8, 425-434.

Srikandakumar, A., Johnson, E.H. and Mahgoub, O. (2003). Effect of heat stress on respiratory rate, rectal temperature and blood chemistry in Omani and Australian Merino sheep. Small Ruminant Res., 49: 193-198.

Tarek, M. M. A. (2002). Comparative study between sheep and goat in their adaptability under Egyptian conditions. Ph.D. Thesis, Fac. Of Agric., Al-A zhar Univ. 
الإستجابات الحرارية لإناث الماعز البلاي والخليط تحت الظروف شبه الحاره.

حسين مصطفى الظاهر 1، عبدالكريم إبراهيم محمد السيد² ، حسن أحمد مصطفى فرغلي 1 و فاطمه الدريس ابراهيم طعيمه 1

1. قسم التطبيقات البيولوجية - شعبة تطبيقات النظائر المشعة - مركز البحوث النووية - هيئة الطاقة الذرية

2.قسم الإنتاج الحيواني -كلية الزراعة - جامعة بنها

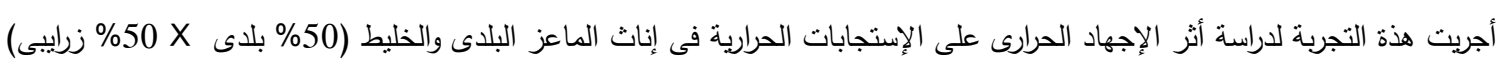
التى نم تربيتها فى منطقة انشاص الصحراوية وذلك خلال دورة الثنبق ومرحلة الحمل وما بعد الولادة تحت ظروف الجه الحت الحار (خلال الفنرة من

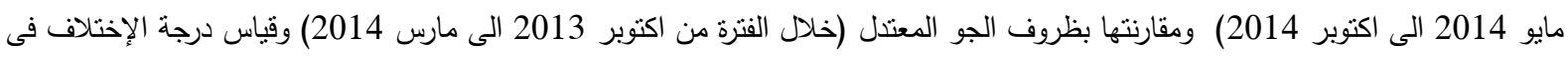
أداء كل من الحيوانات الخليط و البلدى وذلك لتقييم تكيف كل منهما مع ظروف الجو الحار المصريه. وقد استخدم فى هذا البحث 40 انثي ماعز

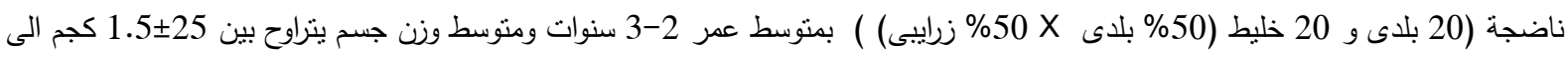
35×6 كجم لكل من البلدى والخليط على الترتيب واستمرت التجربة لموسمين (معتدل وحار). تم وضع الحيوانات في حظائر نصف مفتوحة خلال فترة التجربة وسمح لها بالرعي لمدة خمسة ساعات يومياً علي الأقل. وتم تقدير الإستجابات الحرارية علي الحيوانات متمثلة في درجة حرارة المستقيم والجلد ومعدل التتفس. أوضحت النتائج نقص معنوى فى كل من حرارة المستقيم وحرارة الجلد ومعدل التتفس خلال الموسم معتدل الحراره مقارنة بالموسم الحار خلال دورة الثبق. وأيضا لوحظ نقص فى درجة حرارة المستقيم فى الماعز الخليط مقارنة بالماعز البلدي وذللك خلال المراحل المختلفه من دورة

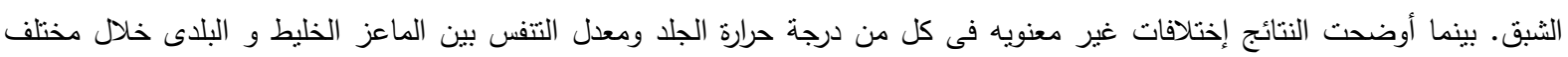
مراحل دورة الثبق. خلال فترة الحمل سجلت إناث الماعز البلدي والخليط إنخفاضا معنويا فى كل من درجة حرفئ حرارة المستقيم و درجة حرارة الجلد ومعدل التنفس خلال مختلف خلال الموسم المعتدل الحرارة مقارنة بالموسم الحار ـ أظهرت إناث الماعز الخليط إنخفاضا ملحوظا فى درجة حرارة المستقيم مقارنة بإناث الماعز البلدى خلال مختلف مراحل فترة الحمل. بينما اظهر كل من درجة حرارة الجلد ومعدل التتفس إختلافات غير معنوية

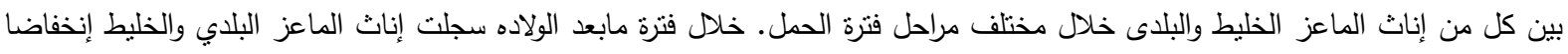
معنويا فى كل من درجة حرارة المستقيم ودرجة حرارة الجلد ومعدل التنفس خلال الموسم المعتدل الحرارة مقارنة بالموسم الحار ـ أظهرت النتائج

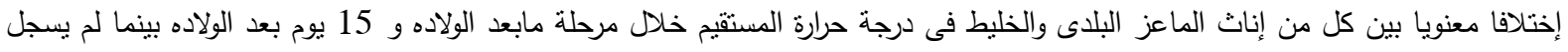

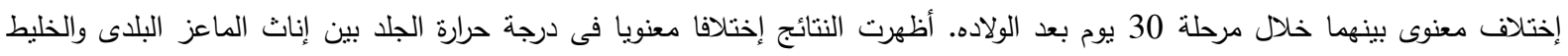

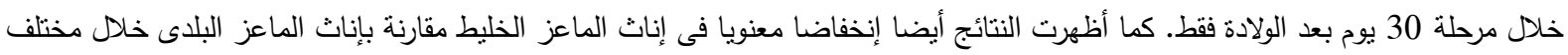
مراحل مابعد الولاده. من نتائج هذه التجربة يمكن استخلاص ان لكل من الموسم و السلالة وكذلك المرحلة الفسيولوجيه التى تمر بها إناث الماعز البلدى والخليط (50\% بلدى X0 X0 زرايبى) تأثنيراً واضحا على درجة حرارة المستقيم والجلد ومعدل التنفس طبقاً لنتائج هذه التجربة. 